\title{
L'espace public dans la photographie d'art du socialisme " réellement existant » : Helga Paris, Ulrich Wüst, Kurt Buchwald
}

Der öffentliche Raum in der fotografischen Kunst des „real existierenden

Sozialismus": Helga Paris, Ulrich Wüst, Kurt Buchwald

Public space in the art photography of "truly existing" socialism: Helga Paris,

Ulrich Wüst, Kurt Buchwald

\section{Emmanuel Béhague}

\section{OpenEdition}

Journals

Édition électronique

URL : http://journals.openedition.org/ceg/1391

DOI : $10.4000 /$ ceg. 1391

ISSN : 2605-8359

Éditeur

Presses Universitaires de Provence

Édition imprimée

Date de publication : 15 juin 2015

Pagination : $31-47$

ISBN : 978-2-85399-993-9

ISSN : 0751-4239

\section{Référence électronique}

Emmanuel Béhague, «L'espace public dans la photographie d'art du socialisme

« réellement existant » : Helga Paris, Ulrich Wüst, Kurt Buchwald », Cahiers d'Études Germaniques [En ligne], 68 | 2015, mis en ligne le 17 décembre 2017, consulté le 02 décembre 2020. URL : http:// journals.openedition.org/ceg/1391 ; DOI : https://doi.org/10.4000/ceg.1391 


\section{L'espace public dans la photographie d'art du socialisme " réellement existant» : Helga Paris, Ulrich Wüst, Kurt Buchwald}

Emmanuel BÉHAGUE

Université de Strasbourg

Niemand hat die Absicht, eine Mauer zu errichten Walter Ulbricht

À travers cette réponse apportée à la journaliste de la Frankfurter Rundschau Annamarie Doherr lors de la conférence de presse du 15 juin 1961, le premier secrétaire du comité central du parti socialiste unifié d'Allemagne (SED) plaçait la construction du mur de Berlin sous le signe du mensonge, mensonge de la dénégation adressé au monde dans un contexte de regain de tension entre l'Est et l'Ouest depuis 1958.

D'autres avatars du mensonge jalonnent l'histoire de la RDA, et en particulier son histoire économique. Ainsi, la correction des chiffres de la production réelle, afin de les faire correspondre aux objectifs du plan, participe de ce qu'on pourrait considérer comme une forme de "mensonge à soi" global, dans lequel sont refoulées les contradictions entre réalité des résultats et prétention idéologique, et que Stefan Wolle décrit non sans une certaine poésie:

\footnotetext{
Nichts galt so, wie es auf dem Papier stand : Weder Theorie, Gesetze, Unterordnungsverhältnisse noch gar Planziffern und Statistik. Trotzdem nahmen sich die Funktionäre ungeheuer wichtig, berauschten sich an den gefälschten Zahlenkolonnen und an fremd klingenden Schlagworten, die geheimnisvoll wie Zauberformeln klangen und sangen wie kleine Kinder im dunklen Wald das Hohelied der Mikroelektronik. ${ }^{1}$
}

Le point de départ de cette contribution est un autre énoncé, célèbre également, à savoir celui qui revient à affirmer, dans la RDA d'Erich Honecker, que le socialisme existe désormais réellement. "Real existierender Sozialismus ${ }^{2} »$ : à travers cette formulation, associée aux années qui suivirent l'arrivée au pouvoir de Honecker, le

1 Stefan Wolle, Die heile Welt der Diktatur. Alltag und Herrschaft in der DDR 1971-1989, Bonn, Bundeszentrale für politische Bildung, 1999, p. 189.

2 Socialisme réellement existant. 
régime mettait un terme aux aléas des réformes économiques entreprises par Walter Ulbricht, mais marquait également la volonté d'un resserrement idéologique qui opposait l'objectif d'un présent à améliorer à un avenir à construire et signalait un tournant pragmatique:

An die Stelle des Ziels, die Utopie in einer großen Kraftanstrengung zu erreichen oder zumindest die "sozialistische Menschengemeinschaft" in kurzer Zeit zu verwirklichen, trat eine ernüchternde Perspektive. Im Mittelpunkt der Theorie und Politik sollten künftig die "realen Prozesse des gesellschaftlichen Lebens" stehen, insbesondere "der Mensch mit seinen materiellen und geistigen Bedürfnissen". ${ }^{3}$

La formulation avait ainsi, selon $\mathrm{S}$. Wolle, des accents défensifs et résignés ${ }^{4}$. Dans cette réorientation idéologique, l'objectif d'une société sans classe dans laquelle se développerait la personnalité socialiste occupait une place centrale, tout en étant également formulée sous la forme d'une "réalité":

Die Konzeption beschrieb dem Anspruch derer, die sie formulierten, zufolge aber immer zugleich die Wirklichkeit, wenn auch eher aus der Perspektive der Entwicklungspotentiale, die sie in ihr sehen wollten. Aus diesem Umstand erklärt sich der unübersehbare Hang zur ideologischen Überhöhung des bereits Erreichten : der Komparativ, ohnehin ein beliebtes marxistisch-leninistisches Stilmittel, nahm seit den siebziger Jahren überhand. ${ }^{5}$

À partir des années 70, les idéologues du SED invoquent donc de manière régulière cette « existence réelle » du socialisme en RDA, qui cherche en outre à compenser son manque de légitimité par le contrôle étroit de la population ${ }^{6}$. De ce point de vue, une telle invocation relève du mensonge politique moderne tel que le définit Hannah Arendt:

Le mensonge politique traditionnel, si manifeste dans l'histoire de la diplomatie et de l'habileté politique, portait d'ordinaire ou bien sur des secrets authentiques - des données qui n'avaient jamais été rendues publiques - ou bien sur des intentions qui, de toute façon, ne possèdent pas le même degré de certitude que des faits accomplis [...]. À l'opposé, les mensonges politiques modernes traitent efficacement de choses qui ne sont aucunement des secrets mais sont connues de pratiquement tout le monde ${ }^{7}$.

Dans cet essai, la philosophe évoque ainsi des phénomènes de réécriture de l'Histoire, donnant pour exemple De Gaulle faisant de la France un des vainqueurs de 1945 ou Adenauer déniant l'ancrage fort de l'idéologie nazie dans la population allemande. De la même manière, le discours affirmant la réussite du socialisme est-allemand relève d'une réécriture du réel. Au socialisme réellement existant s'oppose la "réalité existante" vécue par la population.

Celle-ci, précisément, est l'objet d'une photographie est-allemande dans laquelle la visée documentaire occupe une place importante. Ulrich Domröse et

3 Sigrid Meuschel, Legitimation und Parteiherrschaft in der DDR, Frankfurt a. Main, Suhrkamp, 1992, p. 221-222.

4 Stefan Wolle, DDR, Frankfurt a. Main, Fischer, 2004, p. 101.

5 Meuschel, Legitimation und Parteiherrschaft in der DDR, p. 223.

6 Cf. Ralph JESSEN, «Partei, Staat und "Bündnispartner" : die Herrschaftsmechanismen der SEDDiktatur », in Matthias JudT (dir.), DDR-Geschichte in Dokumenten, Bonn, Bundeszentrale für politische Bildung, 1998, p. 36.

7 Hannah ARENDT, « Vérité et politique », in La crise de la culture, Paris, Gallimard, 1972, p. 321. 
Enno Kaufhold l'associent à une génération intermédiaire, tandis qu'une autre tendance se caractériserait par une prise de distance vis-à-vis de la photographie comme "reproduction" et une proximité avec les milieux de l'art contemporain de la performance et de l'actionnisme ${ }^{8}$. Sans que la subjectivité en soit absente, l'enjeu d'une photographie documentaire réside dans le rendu de la réalité. Dans le contexte d'un état autoritaire, l'acte photographique devient alors un geste de visualisation d'un réel tangible et vécu opposé à l'abstraction du discours idéologique. Matthias Flügge fait de ce geste la raison d'être de la photographie est-allemande qui ne se met pas au service du régime, et dont «les premières caractéristiques sont la sincérité, la prudence et la quête réfléchie d'une vérité visuelle ${ }^{9} »$. Pour ce qu'Ulrich Domröse appelle « straight photography », l'objectif est le rendu exact de ce qui est vu («genaue Wiedergabe des Gesehenen »), les moyens artistiques (« gestalterische Mittel ») y sont subordonnés ${ }^{10}$. L'enjeu devient une résistance contre la déformation idéologiquement motivée du réel:

Die Bilder der straight photography kamen beim Publikum gut an, weil sie das Bedürfnis nach einer vermeintlich realistischen Wirklichkeitswiedergabe und einer emotionalen Ansprache miteinander verbanden. Ihre verhältnismäßig leichte Lesbarkeit begünstigte es, dass ihre Botschaften verstanden und sogar als Beweismittel gegen die nach wie vor herrschende Wirklichkeitsverzerrung in den Medien angesehen werden konnten. ${ }^{11}$

Si un tel geste traverse la photographie d'art, la question n'en demeure pas moins de savoir s'il s'agit d'apporter des preuves. Ici semble en effet s'opérer une identification entre "réalité" et "vérité" qu'il convient d'envisager avec prudence, dans la mesure où elle revient à postuler le principe d'une vérité objective des faits. Par définition, le cadrage définit un hors-champ, et ne circonscrit donc qu'un fragment du réel. En choisissant son sujet, le photographe opère une sélection, et ce seul acte, quel que soit le traitement réservé à l'image à travers les choix esthétiques (pose du sujet, cadrage spécifique, plan, couleur/ noir et blanc...) est une empreinte qui contredit le principe d'une vérité objective. Le principe même d'une subjectivité de l'artiste vient contredire celle-ci: il ne peut y avoir de langage photographique de vérité, mais une pluralité de stratégies esthétiques au sein d'une même démarche qui se donne le réel pour objet.

Afin d'illustrer ces stratégies seront convoqués avec Helga Paris, Ulrich Wüst et Kurt Buchwald trois artistes développant un travail photographique dans les années 80 . Le tertium comparationis sera l'objet de la représentation (le « sujet» dans la terminologie photographique), à savoir l'espace public physique

8 Ulrich Domröse, Enno KaufHold, «Zwischenzeiten. Bilder ostdeutscher Photographen 19871991 », in Franz C. Gundlach (dir.), Zwischenzeiten. Bilder ostdeutscher Photographen 1987-1991, Düsseldorf, Richter Verlag, 1992, p. 7.

9 Matthias FlügGe, « Helga Paris, Christian Borchert », in Foto-Anschlag. Vier Generationen ostdeutscher Fotografen, éd. par Stiftung Haus der Geschichte der Bundesrepublik Deutschland/ Zeitgeschichtliches Forum Leipzig, Leipzig, E. A. Seemann Verlag, 2001, p. 56. « Ihre [der alten Bildern der DDR] ersten Kennzeichen sind Aufrichtigkeit, Behutsamkeit und die zweifelnde Suche nach einer bildnerischen Wahrheit. ».

${ }^{10}$ Ulrich DomRÖSE, « Realität, Engagement, Kritik », in Geschlossene Gesellschaft. Künstlerische Fotografie in der DDR 1945-1898, éd. par la Berlinische Galerie, Berlin, Kerber, p. 20.

${ }^{11}$ Ibid., p. 21. C'est nous qui soulignons. 
(öffentlicher Raum), très présent dans le travail des artistes évoqués. Il s'agira donc tout d'abord de mettre en évidence le lien étroit entre cette notion et la question du mensonge politique moderne. En abordant ensuite les transformations du champ photographique en RDA au cours des années 60 et 70, on s'intéressera au contexte dans lequel devient possible une diversification dans le langage photographique, avant de présenter et d'analyser les travaux des artistes.

\section{Performativité du mensonge et espace public}

Le lien entre mensonge et espace public apparaît dès lors que l'on pense de manière conjointe le discours idéologique et les formes de sa production et de sa diffusion par le biais de la notion de performativité, que nous introduisons ici dans la mesure où elle s'articule de deux manières avec le mensonge. Évoquant la production de discours ou de vérités à dimension "étatique", à partir de l'exemple de la reconnaissance officielle par le Président Jacques Chirac de la culpabilité de l'État français dans la déportation de dizaines de milliers de Juifs et d'autres mesures durant l'Occupation, Jacques Derrida, dans les prolégomènes à son Histoire $d u$ mensonge, met en évidence le caractère performatif de tels objets, non pas de l'acte discursif lui-même, le "mentir », mais de l'objet du discours. Proclamation d'une indépendance, d'une souveraineté, reconnaissance ou non d'une frontière, il existe des objets qui en soi « produisent une vérité »:

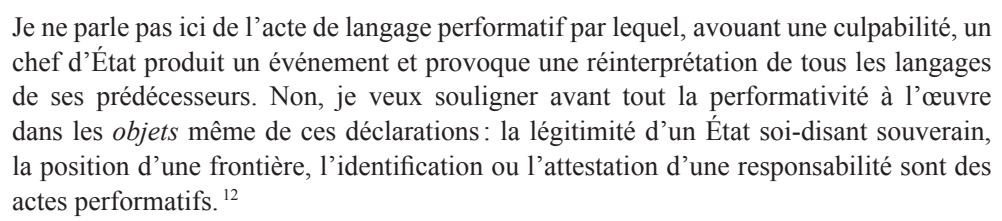

Certes, on objectera que si le socialisme réellement existant est une construction discursive, la culpabilité française est, elle, une réalité; la reconnaître revient donc à rétablir une vérité jusque là occultée. Néanmoins, il s'agit bien pour le régime est-allemand de "faire vérité », pour reprendre ici les termes de Derrida. Du reste, le philosophe élargit le spectre de cette performativité aux " contre-vérités » pour lesquelles «la puissance capitalistico-techno-médiatique» peut produire des «effets de vérité ${ }^{13}$. Il n'existe peut-être pas, dans la RDA des années 70, de puissance « socialisto-techno-médiatique », même si le pouvoir utilise massivement les médias. Il existe néanmoins d'autres moyens de produire de tels «effets de vérité »: faire défiler des chars, des hommes, une jeunesse socialiste, produire une architecture à la fois moderne et sociale, construire un Palais de la République que l'on remplira d'ampoules électriques ( "Erichs Lampenladen »), comme pour attirer sur soi la lumière. Nous avons désormais affaire à une seconde performativité, qui vient doubler celle de l'objet, et touche à la forme de sa production, de sa "mise

\footnotetext{
12 Jacques Derrida, Histoire du mensonge. Prolégomènes, Paris, L’Herne, 2005, p. 65.

13 Ibid., p. 77.
} 
en scène": cette scène, c'est l'espace public physique, dans lequel le collectif doit s'inscrire harmonieusement.

Car il s'agit bien de garantir une visibilité de cette vérité produite. Dans ses Réflexions sur le mensonge, publiées pour la première fois en 1943 et consacrées au lien entre totalitarisme et mensonge, Alexandre Koyré, de manière apparemment paradoxale, associe celui-ci au modèle de la société secrète, contrainte à la dissimulation et à la dénégation pour garantir son existence au sein d'un groupe plus large. Les régimes totalitaires, certes, « opèrent » au contraire « en plein jour »: «Aussi, bien loin de vouloir se fermer, et d'élever une barrière entre eux-mêmes et les autres, leur but, avoué et patent, est-il justement d'absorber tous ces "autres", d'englober et d'embrasser la nation (ou la race) toute entière ${ }^{14}$. » Opérant un retournement qui nous paraît fécond pour une réflexion sur le mensonge politique, Koyré réinterprète néanmoins le rapport entre mensonge et vérité :

\title{
Il est vrai qu'Hitler (ainsi que les autres chefs des pays totalitaires) a annoncé publiquement tout son programme d'action. Mais c'était justement parce qu'il savait qu'il ne serait pas cru par les « autres », que ses déclarations ne seraient pas prises au sérieux par les non- initiés; c'est justement en leur disant la vérité qu'il était sûr de tromper et d'endormir ses adversaires ${ }^{15}$.
}

Ici encore, il est nécessaire de procéder avec prudence dans l'argumentation. En aucune manière, il ne s'agit de mettre sur le même plan le régime nazi et le régime est-allemand, notamment quant à la question du caractère « totalitaire » du système ; par ailleurs, la proclamation du socialisme réellement existant ne relève pas de ce « mensonge au deuxième degré » - selon l'expression de Koyré ${ }^{16}$ - qui s'inscrirait dans la même stratégie. Néanmoins, le régime est-allemand des années 70, du fait même de la véhémence à la fois rhétorique et visuelle avec laquelle il affirme son succès, paraît pouvoir être associé à ce que Koyré appelle les "conspirations en plein jour », qu'il décrit de la manière suivante :

\begin{abstract}
Une conspiration en plein jour - forme nouvelle et curieuse du groupement d'action, propre à l'époque démocratique, à l'époque de la civilisation de masses - n'est pas entourée de menace et n'a donc pas besoin de se dissimuler; bien au contraire, étant obligée d'agir sur les masses, de gagner les masses, d'englober et d'organiser les masses, elle a besoin de paraître à la lumière, et même de concentrer cette lumière sur elle-même et surtout sur ses chefs ${ }^{17}$.
\end{abstract}

Si l'espace public physique est le cadre de l'expression performative du mensonge, l'architecture, véritable fil rouge de l'histoire de la RDA, en modèle les contours, depuis les années de jeunesse de la «meilleure Allemagne » durant lesquelles le programme de reconstruction urbanistique est présenté par la propagande comme le point de départ de la construction de toute une nation ${ }^{18}$, jusqu'aux grands programmes de construction de logements fondés sur le principe de la standardisation

14 Alexandre Koyré, Réflexions sur le mensonge, Paris, Alia, 1998, p. 35.

15 Ibid., p. 36

16 Ibid., p. 37.

17 Ibid., p. 37-38.

18 Joachim PALUTSKI, Architektur in der DDR, Berlin, Reimer Verlag, 2000, p. 44. L'ouvrage constitue une étude particulièrement éclairante sur l'évolution de la politique urbanistique en RDA jusqu'en 1989. 
dans les périphéries urbaines. Depuis les années 50, l'architecture est donc un sujet privilégié tant d'une photographie assumant la fonction d'une illustration affirmative des réalisations du régime, que d'une photographie plus indépendante, à visée " documentaire », à l'instar des travaux d'Arno Fischer, qui dans la série Situation Berlin 1953-1960 témoigne de l'actualité de la ville et des traces de son histoire, en en saisissant les habitants évoluant dans l'espace public ${ }^{19}$. En photographiant les éléments architecturaux, les artistes des années 80 s'inscrivent donc dans une tradition. La production d'un tel langage visuel n'est cependant possible que si le permet l'organisation du champ de la photographie.

\section{L'évolution du champ photographique en RDA}

Le premier constat à faire concernant la photographie en RDA est celui d'une visibilité quasi exclusive de la photographie vantant les mérites du socialisme durant les vingt premières années. Le contrôle exercé par l'État est étroit, ce qui rend presque impossible l'avènement d'une photographie dite documentaire et sociale ${ }^{20}$. La production photographique qui bénéficie des possibilités d'exposer et de publier est avant tout la photo de presse. À celle-ci est associée une fonction illustrative, axée sur la représentation de la société socialiste en devenir, de l'homme socialiste. Malgré tout existe bien une photographie qui se démarque du discours officiel en ce qu'elle se donne pour objet le quotidien est-allemand. Néanmoins, les possibilités d'accéder à une visibilité sont très limitées, et les artistes soucieux de documenter la réalité est-allemande travaillent avant tout «pour le tiroir ») (« für die Schublade ») ou « pour le carton » (« für die Kiste »).

Pour cette photographie se pose la nécessité de s'organiser à travers la formation de groupes, en créant, autant que faire se peut, des espaces et des cadres institutionnels. Le premier de ces espaces est la classe de photographie documentaire à la Hochschule für Grafik und Buchkunst, seul lieu de formation à la photographie en RDA. Y enseignent en particulier, non sans entraves, Evelyn Richter et A. Fischer, qui vont largement influencer la génération suivante et jouer un rôle essentiel dans le développement de la photographie en RDA. À partir du milieu des années 60 sont constitués divers regroupements, dans lesquels les artistes disposent de davantage de possibilités pour développer un style propre et acquérir une relative visibilité. Il faut citer ici les groupes Signum (1965-1969), Jugendfoto Berlin (1969-1979), ou encore le Gruppe DIREKT (1966-1982), constitué autour d'A. Fischer. Cette organisation en collectifs ne dispense cependant pas les artistes d'être rattachés à une organisation officielle, condition nécessaire pour que soit reconnue leur production: il s'agit du Journalistenverband ou du Verband Bildender Künstler. Par ailleurs

19 Voir notamment les photographies choisies dans le catalogue de l'exposition Geschlossene Gesellschaft. Künstlerische Fotografie in der DDR 1945-1989, organisée à la Berlinische Galerie du 5 octobre 2012 au 28 janvier 2013 (cf. note 10).

${ }^{20}$ Bernd LindNER, « Ein Land - Zwei Bildwelten. Fotografie und Öffentlichkeit in der DDR », in Karin Hartewig, Alf LÜDTKe, Die DDR im Bild, Göttingen, Wallstein, p. 189 sq. La synthèse que nous faisons ici de la structuration du champ photographique s'inspire largement de cet article. 
est fondée en 1958 au sein du Kulturbund la Zentrale Kommission für Fotografie, chargée de coordonner le travail dans ce secteur, ce qui implique la définition de l'orientation idéologique, mais aussi le soutien à la jeune création, la mise en place de publications, l'organisation d'expositions ${ }^{21}$.

À partir de la fin des années 70, la photographie d'art va disposer d'une plus grande visibilité. La volonté de fonder un groupe autonome au sein du Verband Bildender Künstler débouche en 1981 sur la création du Arbeitsgruppe Photographie dans laquelle A. Fischer a une part active, ce que l'on peut considérer comme un pas décisif dans l'établissement de la photographie d'art ${ }^{22}$. Les artistes qui en font partie vont dès lors être représentés dans diverses expositions d'art contemporain. Cette reconnaissance se traduit également par l'organisation nouvelle de manifestations d'envergure telles que l'exposition Medium Fotografie à Halle en 1977 ou la 9 exposition d'art de Dresde, avec une section photo indépendante. En 1985 est fondée à Berlin la première galerie de photographie indépendante en RDA, à l'initiative d'U. Domröse et Ralf Herzig; elle restera la seule jusqu'en 1989. Les maisons de la culture de quartier, à partir des années 70, constituent de petits lieux d'exposition, tandis qu'apparaissent peu à peu des galeries autonomes, installées dans des espaces privés ou des ateliers. Celles-ci font du reste l'objet de l'observation attentive de la $S_{t a s i}{ }^{23}$. C'est donc dans ce contexte d'une émergence d'espaces de création que travaillent les artistes dont il est ici question.

\section{Helga Paris}

Née en 1938 à Gollnow, l'actuel Goleniów en Pologne, H. Paris est entrée dans le domaine photographique en autodidacte, puisqu'elle a suivi une formation à la Fachschule für Bekleidung de Berlin avant d'exercer la profession de graphiste à la Deutsche Werbe- und Anzeigengesellschaft. Elle travaille en 1967-68 dans un laboratoire de photographie avant d'intégrer le Verband Bildender Künstler en 1972, tout en finançant son activité artistique par la reproduction d'œuvres d'art pour des catalogues. En 1978, elle connaît sa première exposition individuelle dans le cadre de la Hochschule für Bildende Künste de Dresde. Elle consacre avant tout son travail à l'exploration visuelle de divers milieux sociaux, dans des séries telles que Müllfahrer (1974), Berliner Kneipen (1975), Möbelträger (1975), Altersheim (1980), Berlin-Gürtelstraße (1980), Berliner Jugendliche (1981-82). À cette photographie documentaire vient s'ajouter, à partir de 1981, une réflexion sur l'autoportrait.

La série étudiée dans le cadre de cet article rassemble des clichés de la ville de Halle, et a connu une histoire particulière, qui permet d'en mieux saisir la portée critique. Ce travail n'est pas le fruit d'une commande, mais l'initiative personnelle d'une photographe confrontée "par hasard", parce que sa fille faisait ses études à

\footnotetext{
${ }^{21}$ Monika EIgmüller, Katrin Sonntag, Kerstin ZIEHe, « Biographische Hinweise und Stichworte zur Fotografie in der DDR », in Foto-Anschlag. Vier Generationen ostdeutscher Fotografen, p. 147.

${ }^{22}$ Lindner, « Ein Land - Zwei Bildwelten », p. 196.

${ }^{23}$ Eigmüller, Sonntag, Ziehe, « Biographische Hinweise und Stichworte zur Fotografie in der DDR », p. 144
} 
Halle ${ }^{24}$, au délabrement du centre-ville. Dans cette confrontation, la photographe elle-même souligne la distance qui la sépare du sujet:

Ich habe Halle fotografiert wie eine fremde Stadt in einem fremden Land - Versuch, alles, was ich wissen und verstehen könnte zu vergessen. So, als hätte ich beispielsweise in Rom fotografiert. ${ }^{25}$

C'est précisément ce regard « étranger » sur la ville, révélateur de ce que ses habitants ne voient pas, qui incite les artistes locaux à préparer une exposition intitulée Häuser und Gesichter. Halle 1983-1985 dans la Galerie Marktschlößchen de Halle. Le projet fut tout d'abord validé par le bureau de la section locale du Verein Bildender Künstler, puis, deux semaines avant le vernissage en juin 1986, repoussé. Malgré ses démarches, notamment auprès du Président du Verband, le peintre Willi Sitte, mais aussi des fonctionnaires du Parti et de la ville, l'exposition fut finalement annulée après de longues tergiversations ${ }^{26}$. Elle ne sera finalement présentée qu'en 1990 sous le titre Diva in Grau. Häuser und Gesichter in Halle.

En photographiant un centre-ville délabré, H. Paris le confronte à la politique de construction de logements neufs sous la forme du Plattenbau (immeuble en dalles préfabriquées), telle qu'elle fut développée en RDA à partir du début des années 60, pour connaître rapidement un essor considérable. L'année même où $\mathrm{H}$. Paris photographiait la ville, les bâtiments de logement sous cette forme représentaient $75 \%$ de la construction en $\mathrm{RDA}^{27}$. L'extension de la ville de Halle elle-même commença vers le sud en 1959, mais connut une étape importante avec la construction de la Chemiearbeiterstadt Halle-West, qui devint en 1967 la ville indépendante de Halle-Neustadt, par son ampleur le plus important projet urbanistique de RDA dans les années $60^{28}$. La première pierre en fut posée le 15 juin 1964 par Horst Sindermann, et sa réalisation se fit par étapes. Durant les années 70, le centre-ville fut délaissé, et des parties importantes de celui-ci furent détruites au cours de la décennie suivante. La démarche de la photographe revêt donc une dimension critique: il s'agit d'opposer au projet architectural de la modernité la permanence d'une autre architecture, celle du passé, comme refoulée, mais pourtant bien présente.

Comme l'indique son titre initial, le travail de H. Paris peut être divisé en deux parties: d'une part des clichés de bâtiments, souvent pris en plan large, d'autre part des portraits individuels ou de petits groupes de personnes, photographiées en plan rapproché, le plus souvent en extérieur. Si certains clichés dérogent dans leur composition à cette distinction, elle n'en demeure pas moins essentielle quant au sens à donner au projet: si les habitants de Halle "habitent" bien leur ville, celle-ci n'est pas seulement conçue comme contexte, comme cadre de vie. Il s'agit au contraire, par un jeu de miroir ou de symétrie, de conférer à la ville, à travers ses bâtiments,

${ }^{24}$ Helmut Brade, « Vorwort, 15 Jahre später », in Diva in Grau. Häuser und Gesichter in Halle. Fotografien von Helga Paris, s. 1., Mitteldeutscher Verlag, 2006 [1991], p. 6.

${ }^{25}$ Helga Paris, citée dans : Lindner, « Ein Land - Zwei Bildwelten », p. 198.

26 Pour un historique plus détaillé, voir ibid., p. 197-202.

${ }^{27}$ Christine Hannemann, Die Platte. Industrialisierter Wohnungsbau in der DDR, Berlin, Schelzky und Jeep, 2000, p. 24.

${ }^{28}$ PALUTZKI, Architektur in der DDR, p. 280-289. 
une "vie" telle que celle de ses habitants, ce que le second titre donné à l'exposition, Diva in Grau, semble suggérer. Les maisons de Halle sont autant de figures en gris certes, mais l'identité est soulignée, par-delà la dimension de témoignage du travail photographique sur l'état du centre-ville. Cette valorisation passe en particulier par une verticalité marquée notamment dans les clichés de grands immeubles bourgeois. Le bâtiment apparaît d'autant plus imposant que se trouvent presque toujours à son pied quelques habitants ou quelques véhicules, comme s'il s'agissait d'en suggérer la taille par comparaison.

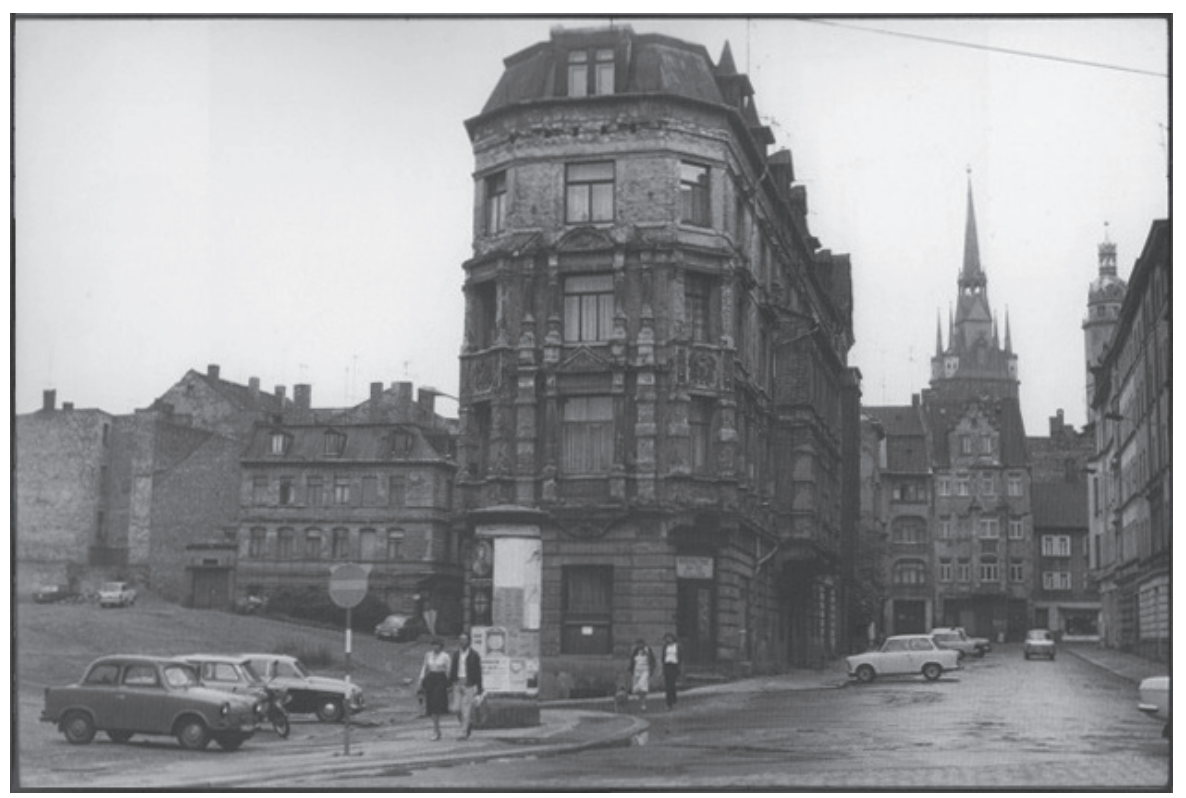

Fig. 1 - Helga Paris, Häuser und Gesichter. (C) Helga Paris, VG Kunst 


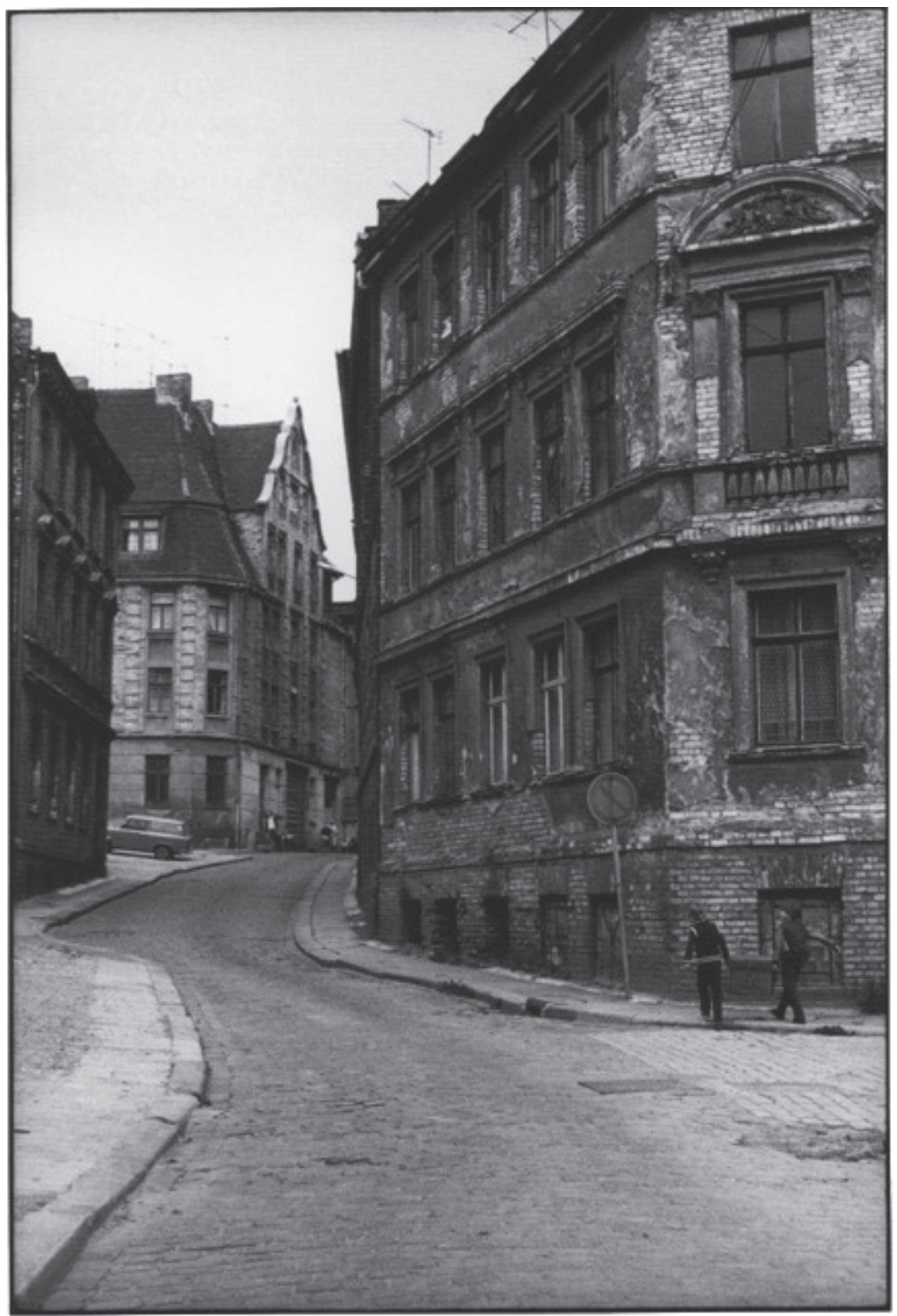

Fig. 2 - Helga Paris, Häuser und Gesichter. (C) Helga Paris, VG Kunst 


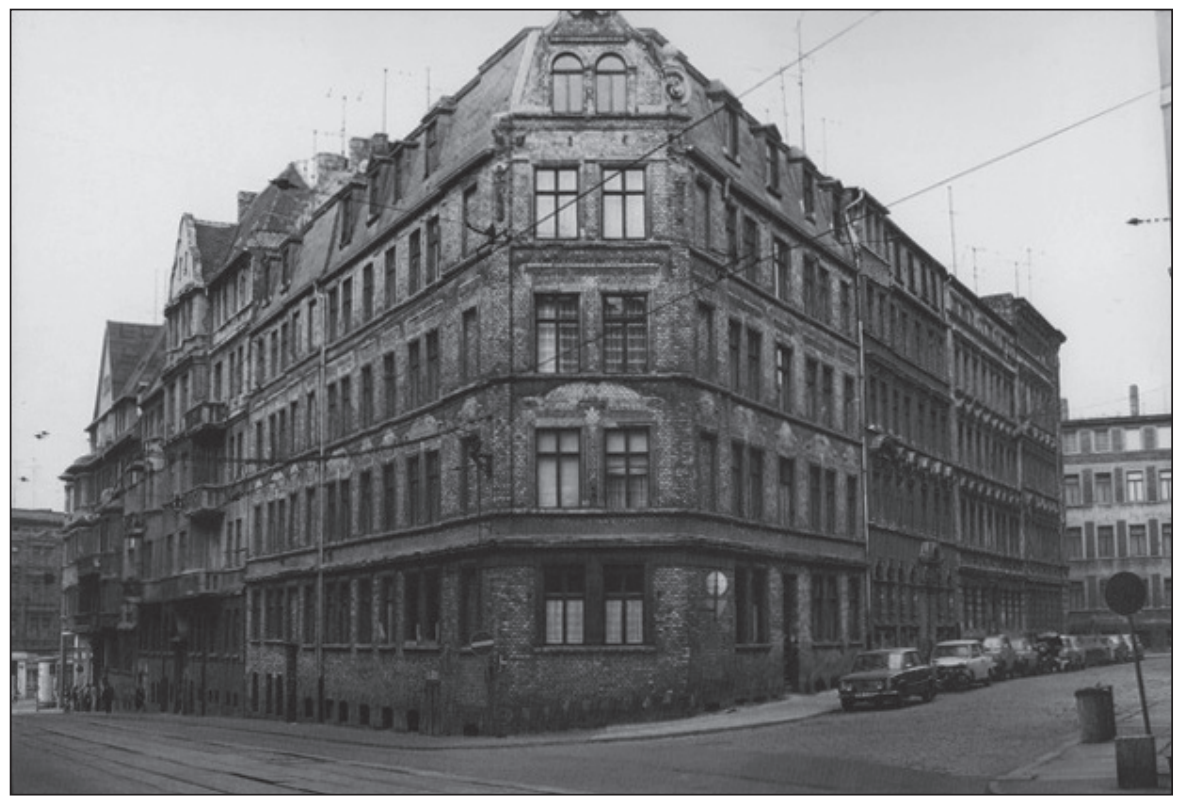

Fig. 3 - Helga Paris, Häuser und Gesichter. (C) Helga Paris, VG Kunst

Par ce regard jeté sur le centre de la ville, H. Paris révèle donc ce qui est délibérément placé "hors-champ". Ce faisant, elle ne confronte pas seulement l'oubli de l'histoire à la modernité, mais oppose en outre une fière verticalité à la standardisation horizontale du Plattenbau. La signification critique d'une telle esthétique apparaît lorsqu'on mesure l'importance de ce mode de construction de masse dans l'histoire urbanistique est-allemande. Dans l'étude qu'elle y consacre, Christine Hanemann fait de celui-ci le produit de la convergence de trois idéologèmes qui sont le postulat de l'égalité sociale, la famille nucléaire socialiste (sozialistische Kleinfamilie) comme cellule modèle de la société nouvelle, et la foi dans le progrès technologique. Comme elle le souligne, le développement de cette architecture conduit précisément à l'abandon d'un urbanisme hérité du passé tel qu'il caractérise le centre historique de Halle:

Aus dieser Sichtweise heraus erklärt sich auch die jahrzehntelange Vernachlässigung der Altbausubstanz und die systematische Reduzierung der Sanierungskapazitäten im Bausektor: Die aus der kapitalistischen Gesellschaft überkommene Stadtstruktur und Wohnbausubstanz wurden als gesellschaftlich überholt betrachtet. ${ }^{29}$

${ }^{29}$ Hanemann, Die Platte. Industrialisierter Wohnungsbau in der DDR, p. 111. 


\section{Ulrich Wüst}

Si on la compare au travail de H. Paris, la série Stadt-Bilder d'U. Wüst, composée de photographies prises entre 1979 et 1987, paraît se situer dans un double rapport de contiguïté et d'opposition. En effet, pour une très grande part de cette série, c'est sur la modernité urbanistique est-allemande que le photographe pose son regard. Mais comme chez Paris, l'architecture en elle-même constitue le sujet photographique, et pas seulement comme “cadre de vie". Né en 1949 à Magdeburg, Wüst n'est devenu photographe qu'après un parcours d'urbaniste. Après des études à la Hochschule für Architektur und Bauwesen de Weimar, il travaille dans ce domaine à partir de 1972 pour la municipalité de Berlin-Est, avant de rompre avec cette activité en 1977 pour se consacrer à la photographie. Il devient membre du Verein Bildender Künstler en 1980, année où il est exposé pour la première fois dans la maison de la culture de Treptow. Il travaille alors en indépendant pour divers magazines tels que Farbe und Raum, Form und Zweck, Bildende Kunst, ou Sonntag.

On peut voir dans cette série, avec U. Domröse, une « critique générale de l'état déprimant du cadre de vie urbain [...], d'un modèle de construction à bon marché et sans imagination en constante expansion, et des nouvelles zones d'habitation surdimensionnées et sans âme en marge des villes ${ }^{30}$ ». L'atténuation des contrastes au profit de nuances de gris renforce l'impression de monotonie et la recherche d'une “objectivité" par le photographe ${ }^{31}$. Celle-ci se traduit également par l'utilisation systématique d'une focale de $50 \mathrm{~mm}$, qui correspond à l'angle de vue "naturel" de l'observateur ${ }^{32}$. Néanmoins, l'analyse du propos photographique et notamment du rapport qu'il suggère entre l'espace urbain et la population censée l'habiter doit sans doute être poussée plus loin. Ainsi, la construction sous la forme de diagonales, l'utilisation soulignée de la perspective, confèrent à l'image une forme de dynamique d'autant plus intéressante qu'elle est paradoxale, dans la mesure où l'espace urbain - et c'est la caractéristique fondamentale de ce travail - est dépeuplé, ou quasiment: il n'y a ici aucune interaction entre l'espace et l'individu. En faisant disparaître les habitants, le photographe remet donc en cause l'espace public comme espace d'échanges et de communication hors de la sphère privée. Vidé de ses habitants, l'espace est vidé de son sens.

${ }^{30}$ Domröse, « Realität, Engagement, Kritik », p. $25:$ « Seine dokumentarisch angelegte Serie StadtBilder [...] ist eine umfassende Kritik an dem deprimierenden Zustand des städtischen Lebensraums [...], an der sich immer mehr ausbreitenden billigen und einfallslosen Bauweise und an den überdimensionierten, seelenlosen Neubaugebieten an den Rändern der Städte ».

31 Ibid., p. 25.

${ }^{32}$ Matthias FLÜGGE, « Die Fotos von Ulricht Wüst aus Berlin-Mitte », http://uinic.de/berlin-mitte/ de/text.html, dernière consultation le 17 mars 2014 : «Das kulturelle Klima als Ausfluß politischer Möglichkeiten erlaubte im Osten für eine Weile den visuellen Gewinn einer Art Wahrheit mit beschränktem Geltungsbereich ». 


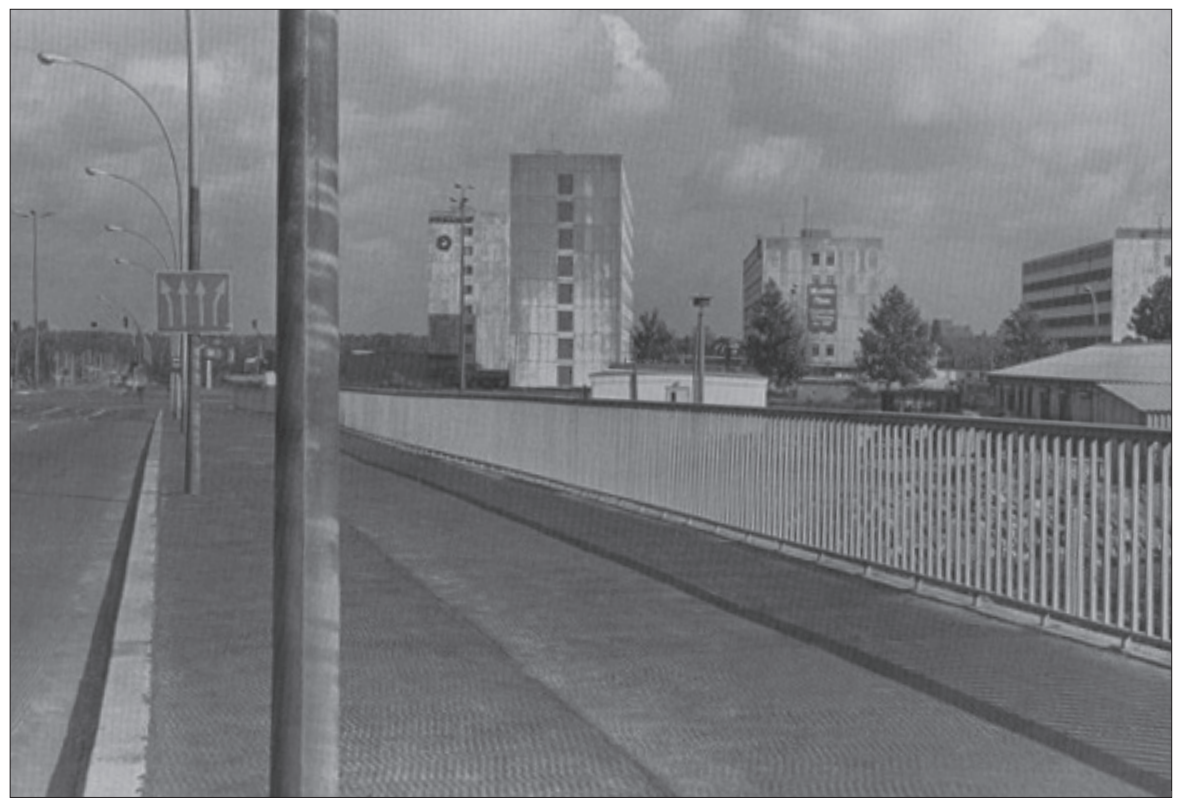

Fig. 4 - Ulrich Wüst, Stadt-Bilder. (C) Ulrich Wüst

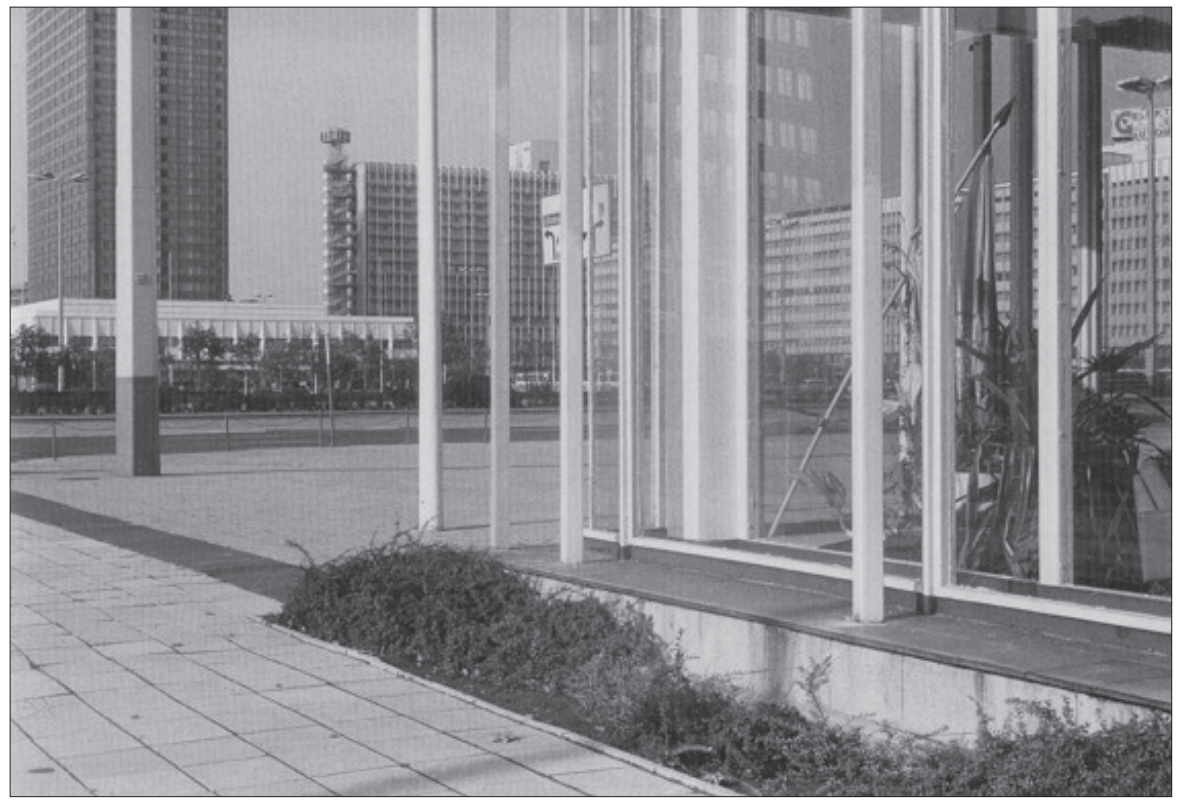

Fig. 5 - Ulrich Wüst, Stadt-Bilder. (C) Ulrich Wüst 


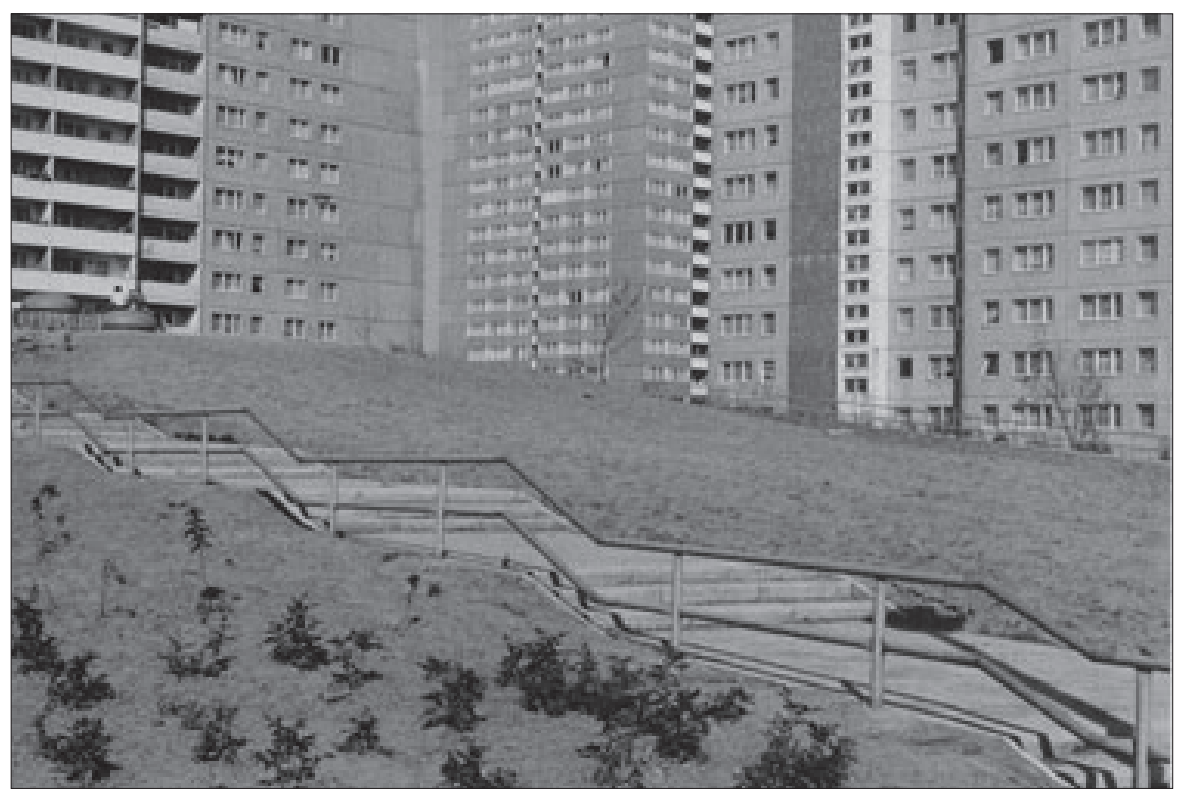

Fig. 6 - Ulrich Wüst, Stadt-Bilder. C) Ulrich Wüst

Chez Wüst, la frontière entre photographie documentaire et photographie subjective devient floue. Le photographe inscrit certes sa démarche dans une volonté de témoigner de la réalité est-allemande, de contribuer, dans le contexte d'une relative libéralisation du champ photographique, au «bénéfice visuel d'une sorte de vérité au champ d'application restreint ${ }^{33} »$. Mais ce regard photographique passe également par une forme de dissimulation, celle d'une population qui pourtant habite bien ses immeubles et traverse bien ces places. Photographier l'espace public "nu" devient la marque d'une subjectivité qui s'affirme au cœur du propos documentaire. Cette articulation entre espace public, "habitants" et subjectivité du photographe caractérise de manière plus poussée encore le travail de K. Buchwald.

\section{Kurt Buchwald}

Né en 1953 à Wittenberg, Buchwald ne dispose pas lui non plus vraiment d'une formation photographique. Après un apprentissage de mécanicien puis des études d'imprimerie à la Technische Hochschule de Karl-Marx-Stadt de 1971 à 1981, il travaille dans ce secteur de 1981 à 1985 à Dresde, avant de s'installer à Berlin-Est pour y devenir artiste indépendant.

Loin de la distance préservée entre le sujet photographique et le regard du photographe chez H. Paris, l'acte photographique en soi constitue chez Buchwald 
une intervention dans l'espace public. Artiste actionniste, il développe un discours métaphotographique en analysant le médium lui-même quant à sa possible manipulation et son instrumentalisation. Le travail intitulé Fotografieren verboten, qui commence en 1988 et s'achève en 2005, peut être évoqué à titre d'exemple: le photographe présente des panneaux d'interdiction de photographier sous la forme d'un pictogramme dans diverses capitales du monde et photographie lui-même les réactions des passants... et des forces de l'ordre dans certains cas.

Dans le cadre de cet article, on s'intéressera plus particulièrement à deux séries complémentaires, dans la mesure où y est repris le même dispositif spécifique entre le sujet photographique et le photographe. Dans Stehplätze-Störplätze, l'artiste constitue un ensemble de vingt photographies prises entre $14 \mathrm{~h}$ et $15 \mathrm{~h}$ le 10 décembre 1984, en différents points de l'Alexanderplatz; en 1986, l'ensemble Ein Tag in Ostberlin reprend le même principe en le déclinant en divers lieux de Berlin-Est: dans le dispositif mis en place, l'artiste se positionne devant l'objectif et gêne la saisie photographique du sujet, ici les passants évoluant dans la ville.

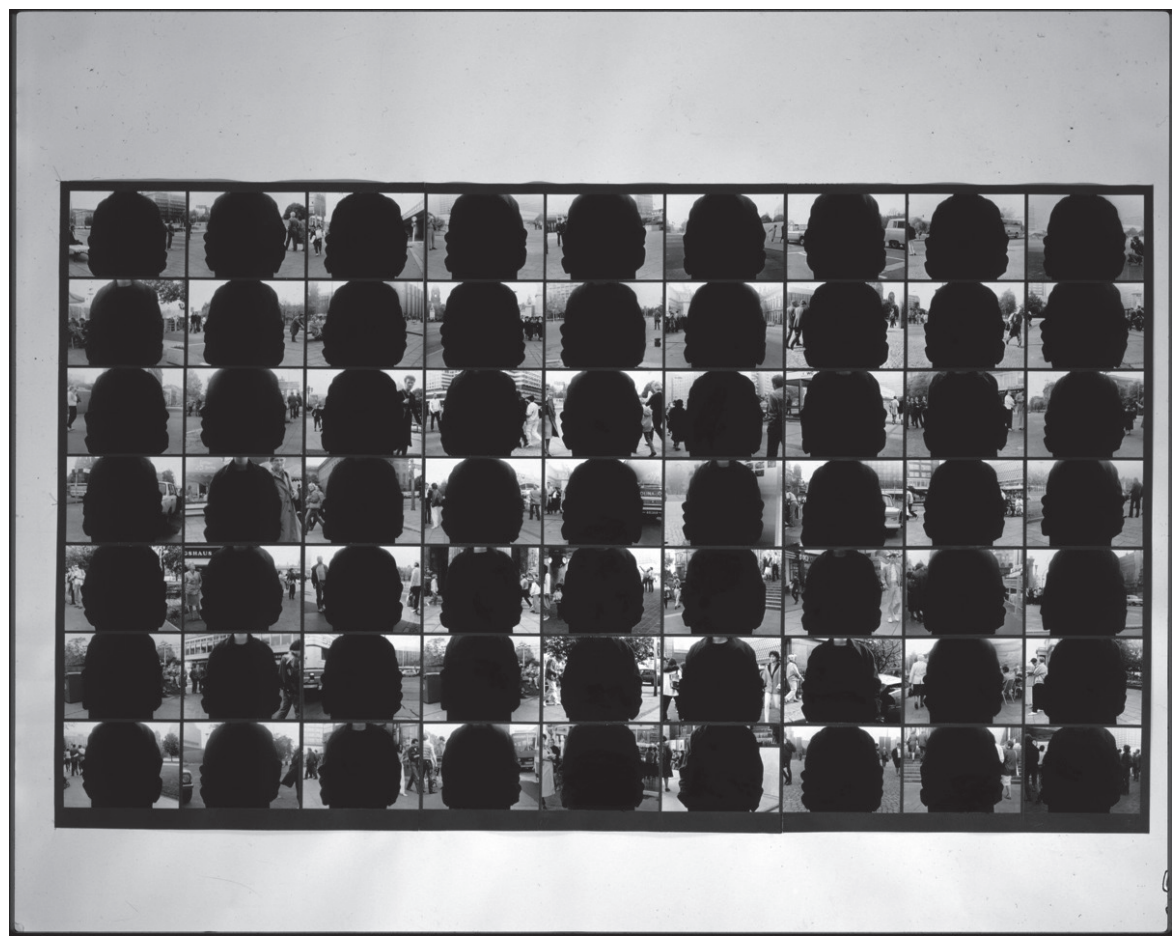

Fig. 7 - Kurt Buchwald, Ein Tag in Ostberlin. Courtesy Galerie David, Bielefeld. (C) Kurt Buchwald 


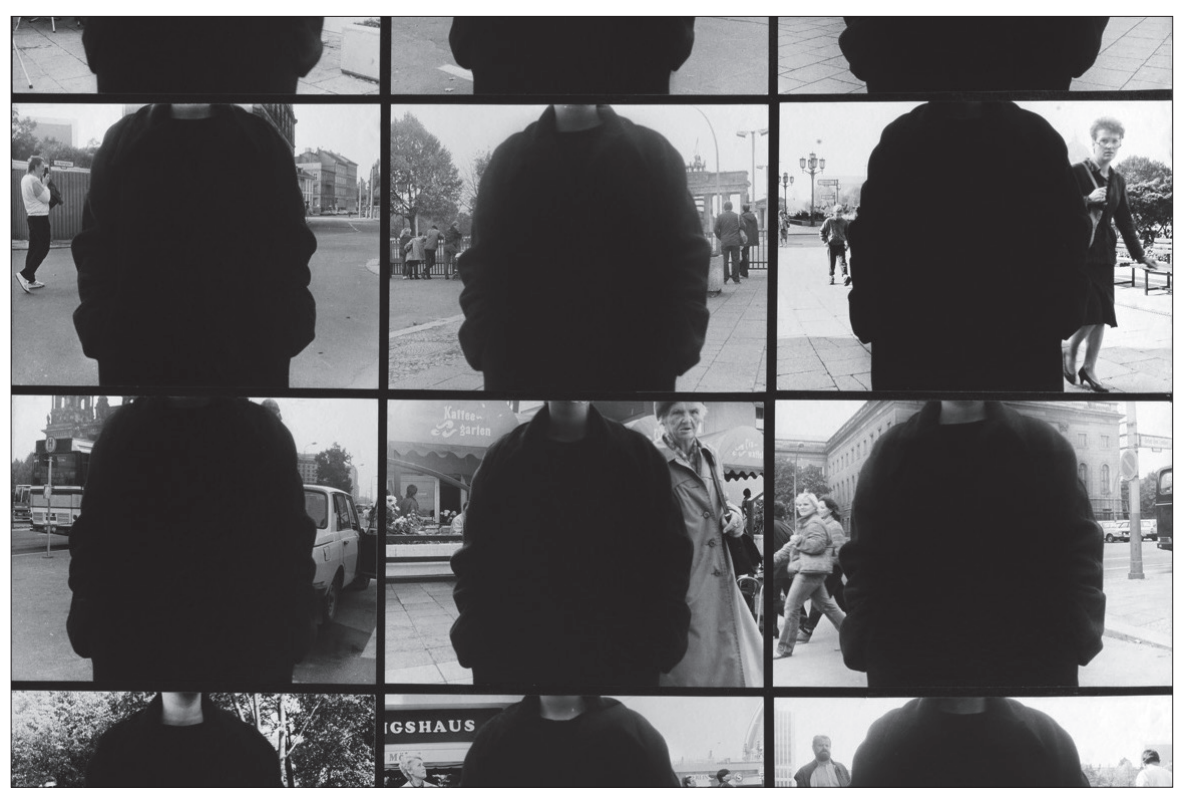

Fig. 8 - Kurt Buchwald, Ein Tag in Ostberlin (Détail). Courtesy Galerie David, Bielefeld. (C) Kurt Buchwald

La photographie est donc « empêchée », pour reprendre le titre d'une exposition de Buchwald à Paris en 1994 à la Galerie Bouqueret-Lebon, ce qui a tout d'abord pour effet paradoxal de voir naître un nouveau rapport d'égalité entre l'observateur et l'objet de l'observation. Regardant la photographie, le spectator cherche à distinguer ce qui demeure visible autour de la surface masquée par le corps de l'operator ${ }^{34}$. Ainsi, leurs positions semblent inversées. À la successivité du phénomène photographique (une photographie est "prise" d'un référent qui lui préexiste, et elle est ensuite "regardée"), Buchwald vient substituer une dynamique dans laquelle l'acte de visée semble être le fait du spectator. La dissimulation du sujet par le photographe est précisément ce qui déclenche l'activité du regard désormais happé dans l'espace public. Mais dans le même temps, les passants photographiés peuvent - il demeure ici une dimension aléatoire qui s'oppose à la construction de l'image chez Paris et Wüst - prendre conscience de la présence du photographe devant son appareil, vers lequel s'oriente alors leur regard. Ainsi naît une forme d'interaction précisément provoquée par l'obstacle que constitue le photographe. Cette interaction

${ }^{34}$ Afin de mieux éclairer le fonctionnement du phénomène photographique dans l'œuvre évoquée, nous employons ici la terminologie proposée par Roland Barthes dans La chambre claire : « J'observai qu'une photo peut être l'objet de trois pratiques (ou de trois émotions, ou de trois intentions) : faire, subir, regarder. L'Operator, c'est le Photographe. Le Spectator, c'est nous tous qui compulsons, dans les journaux, les livres, les albums, les archives, des collections de photos. Et celui ou cela qui est photographié, c'est la cible, le référent, sorte de petit simulacre, d'eidôlon émis par l'objet, que j'appellerais volontiers le Spectrum de la Photographie, parce que ce mot garde à travers sa racine un rapport au « spectacle » et y ajoute cette chose un peu terrible qu'il y a dans toute photographie : le retour du mort. » (Roland BARTHES, La chambre claire, Paris, Cahiers du cinéma, Gallimard/ Seuil, 1980, p. 22-23). 
n'est cependant qu'une possibilité, un hasard qui ne dépendra que de la curiosité du personnage au moment d'être photographié. Par la présence même de l'obstacle est donc préservée une liberté du sujet photographique.

À travers le prisme du sujet photographique que constitue l'espace urbain, la photographie est-allemande contredit donc l'affirmation du « socialisme réellement existant » grâce à un langage visuel multiple qui ne saurait être résumé dans une démarche de restitution d'une "vérité". Conçu comme une photographie du souvenir, le travail de H. Paris oppose à des projets urbanistiques où s'expriment les nouvelles "valeurs" mises en exergue par le régime une mémoire visuelle architecturale saisie au moment de sa disparition. La photographie fait ici œuvre de révélation de ce que veut faire oublier la modernité socialiste.

Les travaux des deux autres artistes présentés ici se distinguent de cette démarche dans la mesure où les approches esthétiques déployées en réponse au mensonge politique d'État semblent relever d'une stratégie qui, dans une forme de dialectique entre le fond et la forme, renvoie à des avatars du mensonge lui-même. En faisant littéralement disparaître les habitants dans l'espace, U. Wüst produit ainsi une illusion. Vide, privé de sa raison d'être, l'espace n'est dès lors précisément plus "public", et devient de ce fait le reflet métaphorique de l'absence de "l'autre" espace public, celui de la sphère intermédiaire entre la sphère privée et l'État (Öffentlichkeit), dans un sens habermassien. On parlera ici d'un subterfuge photographique par lequel l'artiste suggère, à travers la déréalisation du sujet, la réalité d'une absence. Ce faisant, il intervient directement sur ce sujet, une démarche à laquelle paraît s'opposer radicalement celle de K. Buchwald, dont le regard se pose sur l'espace public habité, en mouvement. Mais s'agit-il bien de son regard? En venant s'intercaler devant l'objectif, l'artiste, s'il ne "ment" pas, n'en dissimule pas moins la réalité observée. Il brouille la distribution traditionnelle des cartes de l'art photographique pour mieux faire du fragment capté du réel l'objet non d'une contemplation passive, mais d'un regard actif, car tendu vers son objet.

Alors qu'il s'agit bien, pour une photographie à visée documentaire, de rendre compte de la "réalité" de la société est-allemande par rapport au mensonge idéologique, les démarches analysées montrent combien peuvent être ténues les différences entre les genres telles que les rappellent les observateurs du champ photographique est-allemand invoqués plus haut. Les exemples choisis, sans doute, appellent à être confrontés à d'autres pour rendre mieux compte de sa diversité. Toujours, la subjectivité du regard intervient cependant sur l'objet de ce regard selon des modalités différentes et à des degrés divers. Ce faisant, elle « fait mensonge » au regard du réel, là où le discours politique aspire à « faire réalité ». Un mensonge nécessaire ${ }^{35}$.

\footnotetext{
${ }^{35}$ Nous remercions H. Paris, U. Wüst et K. Buchwald pour l'aimable mise à disposition de leurs photographies.
} 
